\title{
ON THE MECHANISMS OF SPORADIC SOLAR RADIO EMISSION
}

\author{
V. L. GINZBURG \\ Lebedev Physical Institute, Moscow, U.S.S.R. \\ v. V. ZHELEZNIAKOV \\ Lobacheuskii State University, Gorky, U.S.S.R.
}

The mechanism that generates sporadic (nonthermal) solar radio emission, which is of great interest to radio astronomy and sclar physics, is rather obscure. Recently the authors considered this problem [1, 2, 3] and this report gives their summary.

It is convenient to classify the mechanisms as $(a)$ incoherent or coherent, or (b) involving an isotropic plasma or a magnetoactive one. In addition, it is convenient to consider first the conditions of propagation in, and emission of electromagnetic waves from, the solar corona.

\section{1. propagation aNd emission OF electromagnetic waves FROM THE CORONA}

Transverse waves 1 and 2 and a longitudinal plasma wave 3 can propagate in the isotropic plasma. Squares of the refraction indexes for these waves are equal respectively to

$$
\begin{gathered}
n_{1,2}^{2} \simeq \varepsilon \simeq 1-\frac{\omega_{0}^{2}}{\omega^{2}}=1 \equiv \frac{4 \pi e^{2} N}{m \omega^{2}} \\
n_{3}^{2} \simeq \frac{\varepsilon}{\beta_{T}^{2}} \simeq \frac{1-\omega_{0}^{2} / \omega^{2}}{3 V_{T}^{2} / c^{2}}
\end{gathered}
$$

where $N$ is electron concentration, $V_{T}=(k T / m)^{1 / 2}, T$ is kinetic temperature, and $e, m, c, k$ are charge and mass of the electron, light velocity and Boltzmann constant.

In the regions of the corona where $\varepsilon>0$, waves 1 and 2 are slightly absorbed owing to collisions (the role of these collisions is neglected in [1]). The damping of wave 3 takes place, even if the collisions can be neglected; this damping becomes weak only when $\lambda=c / \omega n_{3}$ exceeds Debye's radius $D=$ $\left(k T / 4 \pi e^{2} N\right)^{1 / 2}$.

In the isotropic coronal plasma, electron streams generate only plasma waves; however, we are interested in transverse waves escaping from the corona. In a homogeneous plasma, the transformation of plasma waves into transverse, e.g. radio, waves takes place only owing to scattering on fluctuations of the electron concentration $\delta N=\delta N^{\prime}+\delta N^{\prime \prime}$. Here $\delta N^{\prime}$ represents 
fluctuations of $N$ due to variations of the plasma density, and $\delta N^{\prime \prime}$ the fluctuations of $N$ when ion density practically does not vary. Scattering on fluctuations of $\delta N^{\prime}$ is not followed by a considerable change of frequency (Rayleigh scattering), so the radio wave formed as the result of scattering has the frequency of a scattering plasma wave. Fluctuations $\delta N^{\prime \prime}$ are a combination of plasma waves of fluctuation origin. Radio waves produced by the plasma wave scattering on $\delta N^{\prime \prime}$ fluctuations have the frequency $\omega \sim 2 \omega_{0}$, since the frequency of slightly damping plasma waves is close to $\omega_{0}$.

The whole energy flux of radio waves formed at Rayleigh scattering of the plasma wave on thermal fluctuations of $\delta N^{\prime}$ in the volume $V \sim L^{3}$, is equal to

$$
P^{\prime}(\omega)=\frac{n_{1,2}(\omega) e^{4} N V}{6 m^{2} c^{3}} E_{0}^{2},
$$

where $E_{0}$ is the electric-field amplitude in the plasma wave. The transformation coefficient for the incoherent plasma waves

$$
Q^{\prime} \equiv \frac{P^{\prime}}{S L^{2}} \sim \frac{4 \pi e^{4} N L}{3 m^{2} c^{3} V_{T}},
$$

where

$$
S \simeq \frac{E_{0}^{2}}{8 \pi} \times \frac{d \omega}{d \kappa} \simeq \frac{E_{0}^{2}}{8 \pi} \times \frac{\kappa V_{T}^{2}}{\omega}
$$

is energy flux in the plasma wave. At $N \sim 10^{8} \mathrm{~cm}^{-3}, L \sim 10^{9} \mathrm{~cm}$, and $T \sim 10^{6}{ }^{\circ} \mathrm{K}$ $\left(V_{T} \sim 4 \times 10^{8} \mathrm{~cm} / \mathrm{second}\right)$ one obtains $Q^{\prime} \sim 3 \times 10^{-6}$. For the scattering on $\delta N^{\prime \prime}$ fluctuations, the value of the coefficient of transformation $Q^{\prime \prime} \lesssim Q^{\prime}$ if the scattering plasma waves of a nonthermal origin are absent.

In an inhomogeneous isotropic plasma, besides the transformation due to scattering, a regular transition of plasma waves into radio waves can take place in the regions where $\omega_{0}=\left(4 \pi e^{2} \mathrm{~N} / \mathrm{m}\right)^{1 / 2} \sim \omega$; at the same time the plasma waves must fall at small, but not zero, angles toward the gradient $N$ (see $[4,5])$. For the plasma waves with a wide angular spectrum, the corresponding coefficient of transformation $Q \lesssim 10^{-6}$.

When the influence of a magnetic field is taken into account (since plasma is magnetoactive), the extraordinary and ordinary waves 1 and 2 propagate in the plasma.

The values of $n_{1}^{2}$ and $n_{2}^{2}$ for these waves (they are calculated by the wellknown formulas; see, for example, $[6,7,8]$ ) are given for the corona in Fig. 1 and Fig. $2 a$ for different values of the magnetic field and at the angle $\alpha=15$ degrees between the directions of the field $\vec{H}_{0}$ and wave vector $\vec{K}$. (The case $\alpha=0$ is exceptional, and we do not consider it for simplicity; for $\alpha \neq 0$, Figs. 1 and $2 a$ give qualitative information of the behavior of $n_{1,2}^{2}$ in the corona, when the values of $N$ and $H_{0}$ decrease with increase of the height above the photosphere.) Fig. $2 b$ gives $n_{1,2}^{2}$ and $n_{3}^{2}$ for the same concentration 

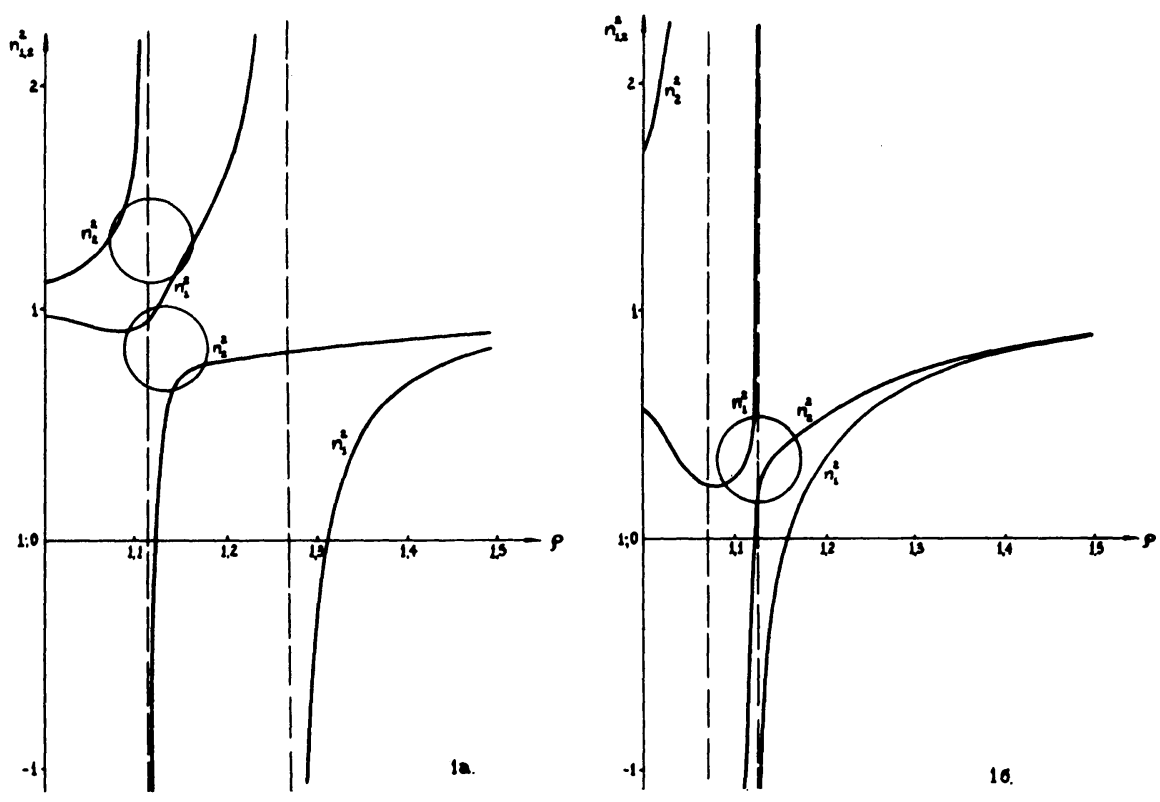

FIG. 1. Dependence of $n_{1,2}{ }^{2}$ on $\rho=R / R_{\odot}\left(R=\right.$ distance from the sun's center, $R_{\odot}=6.95$ $\times 10^{10}=$ photosphere radius $)$. Electron concentration in the corona $N=10^{8}\left(1.55 p^{-6}+\right.$ $\left.2.99 \rho^{-16}\right) \mathrm{cm}^{-3}$; magnetic field strength $H=H_{0}\left[1-h\left(h^{2}+b^{2}\right)^{-t}\right]$ oersteds; $\left(h=R_{0}\right.$ $[\rho-1]=$ height above the photosphere $; b \simeq 3 \times 10^{9} \mathrm{~cm} \mp$ radius of the solar spot). (a) $H_{0}=2500$ oersteds; $(b) H_{0}=250$ oersteds.
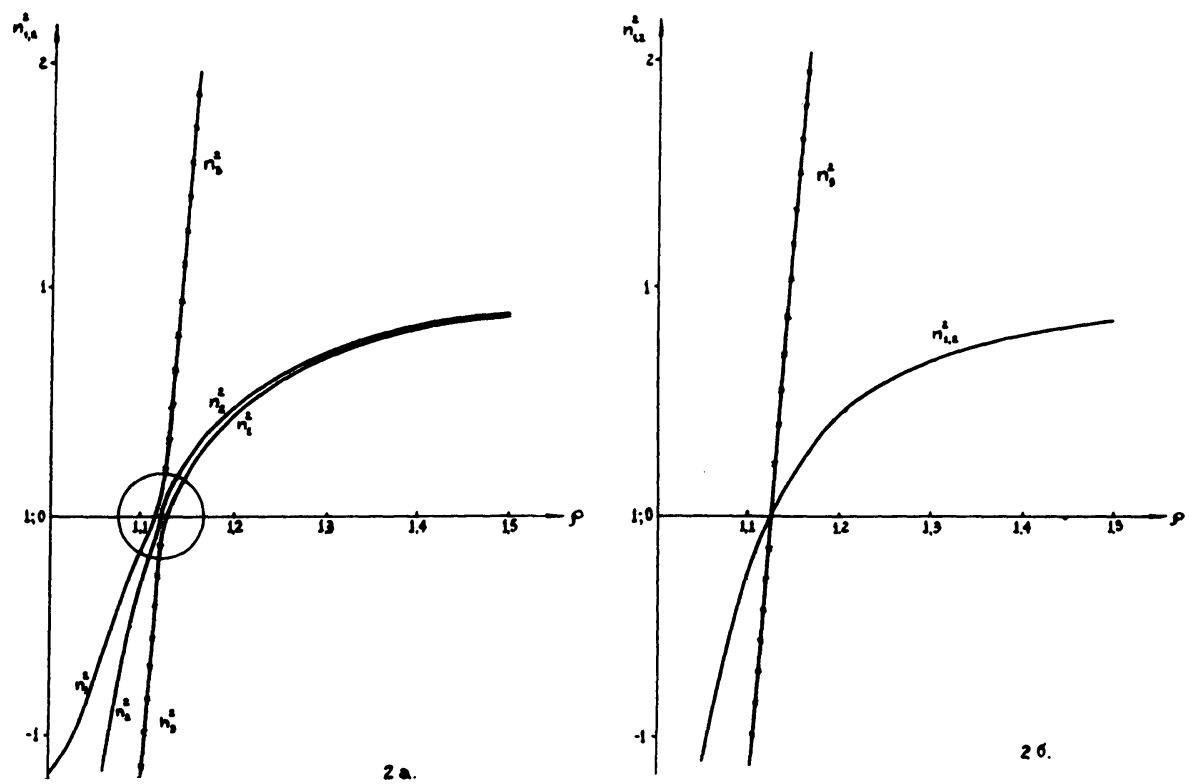

Fig. 2. Dependence of $n_{1,2}{ }^{2}$ on $\rho=R / R_{\odot}$ with the same values of $N$ and $H / H_{0}$ as in Fig. 1 . (a) $H_{0}=25$ oersteds; (b) $H_{0}=0$ oersteds. 
$N\left(R / R_{\odot}\right)$ as in Fig. 1 and Fig. $2 a$ but for zero magnetic field. (Thermal motion is neglected in Figs. 1 and $2 a$; it is taken into account in [8,9]; in the case of Fig. $2 b$, the formulae (1) were used.) It is important that in Fig. $2 a$ a crossed part of the curve in a sufficiently weak field corresponds by all properties to a crossed branch of the curve $n_{3}^{2}$ in Fig. $2 b$.

If electromagnetic waves are generated in the region of the corona where $n_{1,2}^{2}>1$ (see Figs. 1 and $2 a$ ), the problem of the emission output from the magnetoactive coronal plasma is reduced to determining the coefficient of transformation of the wave with $n_{1,2}^{2}>1$ into the waves 1 and 2 capable of propagation out of the corona at large values of $R / R_{\odot}$ In the homogeneous magnetoactive plasma, the output is possible only as a result of scattering, which takes place approximately the same way in the isotropic medium. At the same time, in the inhomogeneous plasma, a regular transition of one normal wave into the other is possible in the regions outlined in Figs. 1 and $2 a$. This transition is especially effective at small angles $\alpha$, and, besides, depends on strength of the magnetic field $H_{0}$ (see $[8,10]$ ). Generally speaking, in the fields $H_{0}>0.25$ oersted, the output of radiation due to scattering plays the main role.

\section{RADIATION IN ISOTROPIC PLASMA}

Bursts of types II and III, which form a considerable part of the sporadic solar radio emission, are unpolarized or, in any case, nearly unpolarized. Taking into account the conditions of radio-wave propagation and output from the corona, one concludes that the magnetic field in the region of generation of bursts of types II and III is weak; according to some estimates $H_{0}<1$ oersted. Under such conditions the plasma in the first approximation can be considered as isotropic.

The frequency drift and some of their other peculiarities lead us to conclude that bursts of types II and III are generated by particle streams. In the isotropic plasma these streams excite the longitudinal waves only. In the case of the incoherent emission the last fact is clear, because the condition of existence of the Vavilov-Čerenkov effect has the form $\beta n_{i} \equiv V_{0} n_{i} / c>1$, and is fulfilled only for the wave 3 , since $n_{1,2}^{2}<1$. The existence of a longitudinal electric field in the plasma wave leads to the stream's instability in the plasma and results in a coherent emission of the plasma waves. We note that from the quantum point of view the instability of the stream in plasma is connected with the fact that this system has a negative absorption (i.e., the induced emission of plasma waves prevails over their absorption). Incoherent and coherent emissions of plasma waves arise simultaneously, but they differ by their dependence on the corresponding parameters; they also differ by their frequency and angular spectra.

The energy of incoherent plasma waves emitted per second by the stream in the frequency range $d \omega$ is equal to

$$
P_{\omega}^{*} d \omega \sim N_{s} L^{3} \frac{3 e^{2} \omega_{0}}{2 V_{0}} \ln \left(1+\frac{2 V_{0}^{2}}{3 V_{T}^{2}}\right) d \omega
$$


where $N$, represents the particle concentration in the stream, $V_{0}$ is their velocity, and $L^{3}$ is the volume of the stream. Hence, one can conclude that an effective temperature of the emitting region, with the area of the order of $L^{2}$, is equal to

$$
T_{\text {eff }}(\omega) \sim \frac{2 \pi^{2} c^{2}}{\omega^{2} k L^{2}} e^{-\tau} Q \frac{\Delta \Omega^{*}}{\Delta \Omega} P_{\omega}^{*}
$$

Here $\Delta \Omega^{*}$ and $\Delta \Omega$ represent solid angles for emission of plasma and radio waves respectively, $Q$ is the transformation coefficient, and $\tau$ the optical thickness for radio waves. Assuming that $Q \sim 3 \times 10^{-6}, \Delta \Omega^{*} \sim \Delta \Omega \sim 1, \tau \sim 4$, $V_{0} \sim 5 \times 10^{\circ} \mathrm{cm} / \mathrm{sec}, L \sim 10^{\circ} \mathrm{cm}, \omega \sim \omega_{0} \sim 2 \pi \times 10^{8} \mathrm{sec}^{-1}$; we obtain $T_{\text {err }}(\omega) \sim$ $4 \times 10^{3} N_{s}$. For the permissible concentration $N_{s} \sim 3 \times 10^{7}$, we have $T_{\text {erf }}(\omega) \sim 10^{11}{ }^{\circ} \mathrm{K}$.

This value determines the upper limit of $T_{\text {erf }}$ since reabsorption of plasma waves in the corona is not taken into account in (5). Estimates show that reabsorption can be rather essential. Unfortunately, we could not take into account the reabsorption entirely and it is possible only to indicate the lower limit of $T_{\text {eff } / \mathrm{mln}}$. For the proton stream $T_{\text {eff } / \mathrm{min}} \sim 2 \times 10^{4}{ }^{\circ} \mathrm{K}$, for the electron stream or mixed quasi-neutral stream the value of $T_{\text {erf } / m i n}$ is lower.

It follows from the estimates given above that the incoherent emission of plasma waves by streams explains* in the main, the appearance of the type III bursts; however, it is quite possible that further consideration of the reabsorption will change the situation.

At the same time the type II bursts cannot be identified with the incoherent emission, since the velocity of these particles $V_{0} \sim 10^{8} \mathrm{~cm} / \mathrm{sec}<V_{T} \sim$ $4 \times 10^{8} \mathrm{~cm} / \mathrm{sec}$ ( $V_{T}$ corresponds to the corona temperature $T \sim 10^{\circ}{ }^{\circ} \mathrm{K}$ ). The point is that when $V_{0}<V_{T}$, a charged particle does not generate the Čerenkov radiation. (This follows from non-fulfillment of the radiation condition $V_{0} n_{3} / c>1$ in the quasihydrodynamic approximation.)

The coherent emission of plasma waves by corpuscular streams explains the peculiarities of the type III bursts, and in all probability, of the type II bursts.

Under certain assumptions (assuming $V_{0}>V_{T}$ and $N_{s}<N$ ), the amplitude of the stationary plasma wave $E_{0} \sim 2 \times 10^{6} N_{s}^{4} / N^{4}$ if $\omega \sim \omega_{0} \sim 2 \pi \times 10^{8} \mathrm{sec}^{-1}$, $V_{0} \sim 5 \times 10^{\circ} \mathrm{cm} / \mathrm{sec}^{-1}, T_{s} \sim T \sim 10^{\circ}{ }^{\circ} \mathrm{K}$; here $T_{s}$ and $T$ stand for temperature in the stream and in the corona, respectively. Hence, for the effective temperature of radio waves, one obtains

$$
T_{\text {orf }}(\omega) \sim \frac{4 \pi^{3} c^{2}}{\omega^{2} k L} \frac{e^{-\tau}}{\Delta \omega \Delta \Omega} P^{\prime}(\omega) \sim 10^{-5} P^{\prime}(\omega) \sim 10^{20} N_{s}^{8} / N^{8}
$$

where $P^{\prime}(\omega)$ is determined by the formula (2) with $n_{1,2}(\omega) \sim 0.1$; in (6) it is also written $L \sim 10^{\circ} \mathrm{cm}, N \sim 10^{8}$ electrons $/ \mathrm{cm}^{3}, \tau \sim 4, \Delta \Omega \sim 0.1$, and it is as-

\footnotetext{
* In incoherent radiation, the appearance of a harmonic with the frequency of $2 \omega$ can be connected with the scattering on fluctuations $\delta N^{\prime \prime}$.
} 
sumed that the width of the spectral band occupied by the burst $\Delta \omega \sim 0.1 \omega \sim$ $2 \pi \times 10^{7} \mathrm{sec}^{-1}$. Thus, at $N_{s} \sim 2 \times 10^{-2} N \sim 2 \times 10^{6} \mathrm{~cm}^{-3}, T_{\text {eff }} \sim 3 \times 10^{12}{ }^{\circ} \mathrm{K}$, which corresponds to the value $T_{\text {erro }} \sim 10^{\circ}{ }^{\circ} \mathrm{K}$ related to the whole solar disk. The last value is sufficient to explain the type III bursts.

A new quantitative calculation is needed for the type II bursts, since in this case the particle velocity in the stream $V_{0}<V_{T}$. However, one can suppose that the coherent radiation also explains the peculiarities of the type II bursts. The appearance of a harmonic in the burst spectrum is easily explained by a nonlinear character of oscillations in the stationary plasma wave. This does not exclude, however, the harmonic from being connected with the output conditions and depending on plasma-wave scattering.

\section{RADIATION IN MAGNETOACTIVE PLASMa}

Since the bursts of type $I$ and the high-level radiation above the spots are polarized, the generation of these components must be considered by taking into account the influence of the magnetic field. In this case, the incoherent emission of moving particles can be divided into the Vavilov-Cerenkov radiation and the magnetobremsstrahlung (synchrotron) radiation connected with the rotation of fast electrons around the lines of force with the frequency $\omega^{*}=\omega_{H} \sqrt{1-\beta^{2}}=\left(e H_{0} / m c\right)\left(m c^{2} / E\right)$. Magnetobremsstrahlung radiation is generated in the region $n_{i}>1$ and also in the layers where $n_{i}<1$. Therefore, as one can see from Figs. 1 and 2 the emission of this radiation from the corona is not a difficult question.

High-level radiation above the spots can be of a magnetobremsstrahlung origin $[5,11,12]$. In order to explain the observed level of radiation, it is sufficient to suppose that in the magnetic field above the spots we have electrons with the energy $E \sim 10^{6} \mathrm{eV}$ and concentration $N \sim 1$ electrons $/ \mathrm{cm}^{3}$. The mechanism of electron acceleration up to the energies $E \sim 10^{6} \mathrm{eV}$ is briefly discussed in [5]. At the same time the problem of the magnetobremsstrahlung mechanism is not yet sufficiently clear, since the model taken in [12] needs modification (see [5]). For this reason it is difficult to say whether it is possible to provide, on the basis of the magnetobremsstrahlung mechanism, the predominance of the ordinary component in the high-level radiation.

It is equally impossible to explain the bursts of the type I by magnetobremsstrahlung radiation of electrons, since there is no reason to assume that emitting particles can considerably change their energy in the times $\lesssim 1^{\mathrm{s}}$ characteristic of the bursts of type I. The bursts of types II and III also cannot be connected with the magnetobremsstrahlung radiation (at any rate, if one assumes electrons to be weakly relativistic, which is necessary to explain harmonics). In [13], where the magnetobremsstrahlung mechanism of the outburst radiation is proposed, the reabsorption, which radically changes the conclusions (see [5]), was not taken into account.

The Vavilov-Cerenkov effect is possible only in those regions where $\beta n_{1,2}>1$. It is clear from Figs. 1 and 2 that the emission of this radiation is complicated and, in all probability, the coefficient of transformation $Q$ does 
not exceed the above-mentioned values for the isotropic plasma. In addition, excluding the case of weak fields $H_{0}<1$ oersted, mainly the ordinary wave will escape. Also, it is impossible to explain the type I bursts on the basis of this mechanism (for the same reason as in the case of the magnetobremsstrahlung radiation). Thus, the Vavilov-Čerenkov effect in the fields $H_{0}>1$ oersted can generally lead to high-level radiation only, but also it plays a lesser role than in the magnetobremsstrahlung radiation.* In a weak field the Vavilov-Čerenkov effect is, as a matter of fact, equivalent to the radiation of plasma waves in the isotropic plasma (see section 1 and Figs. $2 a$ and $b$ ). The connection of incoherent plasma waves with the bursts of types II and III has been already considered above. ${ }^{\dagger}$

Generally speaking, the stream of charged particles moving in the magnetoactive plasma is unstable, bringing about the coherent radiation of ordinary and extraordinary waves. At $\alpha \neq 0$ a longitudinal electric field exists in these waves, which causes grouping of radiating particles (as in the case of longitudinal waves in the isotropic plasma). If the magnetic field is weak (roughly speaking $H_{0}<1$ oersted), this coherent radiation is practically identical to the coherent radiation of plasma waves considered in section 2 when it was applied to the bursts of types II and III. In stronger fields the coherent radiation is emitted from the corona mainly as ordinary waves; therefore, it can only be connected with high-level radiation and also with the type I bursts. These bursts are generated during the damping of free oscillations in the coronal plasma. Estimates show that to produce the observed flux of radiation of the type I bursts, we must have free-plasma oscillations in the corona with the amplitude $E \sim 10^{-1}$ case units. The excitation mechanism of such oscillations is, however, obscure (perhaps shock-magnetohydrodynamical waves play the role of an exciting agent). ${ }^{\ddagger}$

In [16] (see also [5]) the mechanism of free oscillations of the ionospheric plasma is put forward to explain the short sporadic type I bursts emitted by Jupiter and Venus.

In [14] it was assumed that type I bursts are caused by the magnetobremsstrahlung radiation of the system of electron packets (a size of every packet $l<\lambda)$. The electrons of every such packet give the coherent radiation, but a total radiation of the whole system consists of the radiations of separate

* The power of the magnetobremsstrahlung radiation and Črenkov radiation under the considered conditions is, roughly speaking, of the same order. Therefore, the role of the Cerenkov radiation is depressed owing to the small efficiency of its output from the corona. It is possible, however, that this efficiency considerably increases owing to the scattering of the Cerenkov waves on the other non-equilibrium waves of the same origin.

† In the paper [15] at the discussion of the Cerenkov radiation, reabsorption in the corona was not considered, and the output conditions were ignored. Furthermore, the attempt [15] to connect radiation on the frequencies $\omega$ and $2 \omega$ with the frequencies $\omega_{0}$ and $\omega_{H}$ seems to be rather artificial.

\$ The attempt made in [17] to explain excitation of plasma waves by instability of the shock-wave front is groundless (see [5]). 
packets (i.e., the electron phases in different packets have a random distribution). It is clear, however, that such conditions cannot take place in the solar corona, where a length of the free path $l_{\text {free }}$ exceeds by 4 to 6 orders the wavelength of the sporadic radiation in the corona $\lambda \sim 10^{2}$ to $10^{4} \mathrm{~cm}$. Indeed, the appearance of packets with $l \lesssim 10^{2}$ to $10^{4} \mathrm{~cm}$ in the plasma with $l_{\text {rree }} \sim 10^{8} \mathrm{~cm}$, and also the existence of definite phase relations, which provides coherency in the region with a characteristic size of $l$, seem to be doubtful. Moreover, if the packets were produced, they would essentially change their form during the time of the order of $10^{-7}-10^{-5}$ seconds. (This time is sufficient for electrons with a velocity $v_{T} \sim 4 \times 10^{8} \mathrm{~cm} / \mathrm{second}$ to pass along the magnetic field a distance of the order of $\lambda$ ). We see also that the increase of radiation intensity of every packet owing to coherency is compensated by the corresponding increase of its absorption. Therefore, the resulting radiation in a sufficiently thick layer will be the same as for the the system of incoherent electrons.

\section{CONCLUSION}

Summing up, we can say that already the general points of the theory of the sporadic solar radio emission seem to be clear. At the same time, a number of important questions and details must be considered. For a further development of the theory, it is necessary: (1) to determine the polarization of the magnetobremsstrahlung high-level radiation above the spots; (2) to find the mechanism of excitation of plasma oscillations leading to the appearance of type I bursts; (3) to take more completely into account the reabsorption for the incoherent radiation; (4) to calculate the generation mechanism of the type II bursts by the streams of slow particles; and (5) to consider the stable nonlinear plasma oscillations in order to determine the intensities of harmonics. In addition, certainly, there are a number of other questions closely connected with the problem of the sporadic solar radio emission, the most important of which seems to be the mechanism of acceleration of fast particles in the corona.

\section{REFERENCES}

[1] Ginzburg, V. L., and Zhelezniakov, V. V. A. Zh. 35, 694, 1958.

[2] Ginzburg, V. L., and Zhelezniakov, V. V. A. Zh. (In press).

[3] Ginzburg, V. L., and Zhelezniakov, V. V. A. Z h. (In press).

[4] Zhelezniakov, V. V. Radio Engineering and Electronics, U.S.S.R. 1, 840, 1956.

[5] Zhelezniakov, V. V. Usp. Fiz. Nauk, 64, 113, 1958.

[6] Ginzburg, V. L. A. Zh. 26, 84, 1949.

[7] Al'pert, Ia. L., Ginzburg, V. L., and Fainberg, E. L. Radio Wave Propagation. Moscow, 1953.

[8 ] Gershman, B. N., Ginzburg, V. L., and Denissov, N. G. Usp. Fiz. Nauk,61, 561, 1957.

[ 9] Ginzburg, V. L. Zh. Eksp. Teor. Fiz. 34, 1593, 1958.

[10] Zhelezniakov, V. V. in Radiophysics, Proceedings of Higher Schools, U.S.S.R. (In press.) 
[11] Getmantsev, G. G., and Ginzburg, V. L. Dokl. Akad. Nauk S.S.S.R. 87, 187, 1952.

[12] Gershman, B. N., and Zhelezniakov, V. V. in Transactions of the Fifth Conference on Cosmogonical Questions. Moscow (Acad. Sci. U.S.S.R. Press), 1956.

[13] Kruse, U. E., Marshall, L., and Platt, J. R. Ap. J. 124, 601, 1956.

[14] Takakura, T. Publ. Astr. Soc. Japan, 8, 182, 1956.

[15] Marshall, L. Ap. J. 124, 469, 1956.

[16] Zhelezniakov, V. V. A. Zh. No. 2, 1958.

[17] Sen, H. K. Aust. J. Phys. 7, 30, 1954.

Note added in proof: In coronal regions, where the frequency $\omega$ is near $8 \omega_{H}(8=$ $1,2,3 \cdots ; \omega_{H}=e H_{0} / \mathrm{mc}$ ), resonance absorption unconnected with collisions takes place. Due to this absorption, attenuation of ordinary and extraordinary waves (at $\alpha \neq 0$ ) occurs when these waves pass through layers with $8 \omega_{H} \simeq \omega$. The optical thickness of this layer, according to preliminary estimates, is of the order $\tau_{1,2}(8=1) \sim \tau_{1,2}(8=2) \sim 10^{6}$, $\tau_{1,2}(8=3) \sim 60, \tau_{1,2}(8=4) \sim 7 \times 10^{-2}$, etc. Owing to the existence of such nontransparent layers at the levels $\omega \simeq 8 \omega_{H}(8=1,2,3)$, it is impossible in some cases for the waves to escape from the inner corona. This seems to us to be very important $[2,3]$. 